\title{
TCP-Aware Channel Allocation in CDMA Networks
}

\author{
Majid Ghaderi, Member, IEEE, Ashwin Sridharan, Member, IEEE, Hui Zang, Senior Member, IEEE, \\ Don Towsley, Fellow, IEEE, and Rene Cruz, Fellow, IEEE
}

\begin{abstract}
This paper explores the use of rate adaptation in cellular networks to maximize throughput of long-lived TCP sessions. Modern cellular networks incorporate RF technology that allows them to dynamically vary the wireless channel rate in response to user demands and channel conditions. However, the set of data rates as well as the scheduler's rate adaptation policy are typically chosen to maximize the throughput of inelastic connections. We focus on the problem of maximizing the throughput of TCP connections and propose a joint optimization of MAC and physical layer parameters with respect to TCP sending rate. In particular, we propose a simple TCP-aware channel scheduler that adapts the wireless channel rate to changes in the TCP sending rate and explore its performance for both single and multiple concurrent sessions. In the case of a single TCP session, we develop a fluid model of its steady-state behavior in such a system that adapts between two channel rates. The accuracy of the model, its utility in selecting optimal rates as well as the performance of systems with up to three channel rates are explored with $n s-2$ simulations. Our results indicate that a two-rate scheme improves TCP throughput by 15 to 20 percent over a system that does not exploit rate adaptation and that little additional benefit accrues from the addition of a third channel rate. Finally, we extend the framework to scenarios where bandwidth is shared by multiple TCP sessions. We propose two channel allocation algorithms, one relying on detailed TCP state information, the other not, and explore their performance through simulation. Our results indicate that TCP throughput is relatively insensitive to either channel allocation algorithm, and adaptive rate variation is the dominant factor in performance.
\end{abstract}

Index Terms-Wireless networks, TCP throughput, rate adaptation, cross-layer optimization, scheduling.

\section{INTRODUCTION}

$\mathrm{M}$ ODERN digital communication technologies combined with powerful mobile processors now allow wireless channel schedulers in cellular networks to rapidly change the allocated channel resources in response to channel conditions as well as user demands. This is achieved by changing parameters (and combinations thereof) such as the coding rate, spreading factor, modulation scheme, and link-layer retransmission rate. For ease of exposition, we shall refer to these jointly as RF control variables. These variables essentially trade off data rates for improved frame error rates (FERs) and vice versa.

Cellular networks typically specify various combinations of the RF control variables that result in a set of allowed data rates and corresponding FER. The RF scheduler dynamically assigns rates from this allowed set based on its rate adaptation policy. For example, in the CDMA2000 1xRTT network [1], the scheduler can dynamically transition between five different data rates during a mobile's session in response to buffer content and channel conditions

- M. Ghaderi is with the Department of Computer Science, University of Calgary, Calgary, AB T2N 1n4, Canada. E-mail: mghaderi@ucalgary.ca.

- A. Sridharan and H. Zang are with Sprint, 1 Adrian Court, Burlingame, CA 94010. E-mail: \{ashwin.sridharan, hui.zang\}@sprint.com.

- D. Towsley is with the Department of Computer Science, University of Massachusetts, Amherst, MA 01003-4610. E-mail: towsley@cs.umass.edu.

- R. Cruz is with the Department of Electrical and Computer Engineering, University of California, San Diego, La Jolla, CA 92093-0407.

E-mail: rcruz@ucsd.edu.

Manuscript received 7 Feb. 2007; revised 1 Dec. 2007; accepted 25 Apr. 2008; published online 14 May 2008.

For information on obtaining reprints of this article, please send e-mail to: tmc@computer.org, and reference IEEECS Log Number TMC-0044-0207.

Digital Object Identifier no. 10.1109/TMC.2008.81. by varying the spreading factor through the Walsh code ${ }^{1}$ length. A shorter Walsh code lowers the spreading factor which results in higher data rates. However, a shorter code results in lower Signal to Interference and Noise Ratio (SINR) and supports fewer users simultaneously.

In practice, the aforementioned parameters as well as the scheduler's rate adaptation policy are chosen to optimize the raw physical layer goodput of a user. The set of data rates is obtained by choosing a combination of the RF control variables that produces the highest channel data rate under a particular channel condition ${ }^{2}$ for a target FER. Similarly, scheduling policies typically assign the highest possible data rate allowed (for a given channel condition) from the set of given data rates that can clear the buffer backlog ${ }^{3}$ (see [2] for a detailed characterization of this behavior). While ideal for inelastic constant rate applications, this resource allocation methodology can produce suboptimal performance of elastic applications and protocols, in particular TCP, that adapt their rate in response to feedback from the receiver.

As is well known, TCP, by far the most dominant transport protocol, uses an additive increase multiplicative decrease (AIMD) algorithm that gradually increases its transmission rate based on receiver feedback and rapidly throttles back when it perceives losses (either due to congestion or channel errors). Given this complex relation between TCP throughput and the channel transmission and

1. In CDMA systems, the Walsh code is an orthogonal code used to identify each user and mitigate interference from other users.

2. A channel condition is defined by a particular range of SINR values and is a function of fading, interference, etc.

3. The CDMA2000 1xRTT is an example of a commercial system with such features. 
loss rate, the same trade-off in channel capacity and FER that works for inelastic traffic may yield data rates and FERs that degrade TCP throughput. Similarly, a scheduler's rate adaptation policy that always aims to clear buffer backlog can be suboptimal for TCP. For example, when the TCP source has a small window and is ramping up its rate, it is very sensitive to losses but not to the assigned channel rate. In such a state, if the RF scheduler allocates a high channel rate at the expense of a high FER (perhaps due to a sudden accumulation of buffer backlog as a result of jitter in the network), the TCP source cannot fully utilize the high rate and, in fact, may drop its window or time out due to channel errors. Conversely, for large windows (high TCP sending rates), it may be advisable to allocate high channel rates even at the expense of high bit error rates, since a low channel rate will inevitably result in packet loss due to congestion. On a related note, a previous study found that sharp bandwidth oscillations induced by rate adaptation of the RF scheduler in CDMA networks that are agnostic to TCP result in throughput degradation [3].

Finally, as noted previously, in current CDMA networks (e.g., CDMA2000 1xRTT), the high-rate channels are achieved by reducing the spreading factor of the orthogonal Walsh codes. This essentially implies that only few users can simultaneously share the high-rate channels at any given time, simply because of the shorter code length. In particular, the higher the channel rate, the fewer the number of concurrent users that can be supported. Thus, selection as well as allocation of high-rate channels must be made in a judicious fashion that not only accounts for TCP behavior but is also fair across users.

The above discussion clearly motivates the case for TCP-aware dynamic rate allocation as a means to increase throughput of TCP sessions over wireless channels. ${ }^{4}$ In order to achieve this objective, such a scheduler should be able to 1) choose control variables such as coding rate that yield the optimal set of data rates (and corresponding FERs) from the perspective of TCP throughput and 2) set channel rates in a manner that is cognizant of TCP dynamics. The proposal of a simple scheduler that captures the aforementioned properties and its analysis form the main objective of this paper.

Specifically, we propose a wireless channel scheduler that allocates different channel rates from a set of optimized rates to TCP sessions in response to their sending rates. In the context of a single TCP session, we develop a model to compute its long-term throughput under such a scheduler and use it for joint optimization of control variables, e.g., spreading factor, to compute the set of optimal channel rates that are used by the scheduler. We extend the rate allocation framework to incorporate the presence of multiple TCP sessions. In particular, given a set of channel rates that are computed by the single flow model, we study different mechanisms for allocating these channel rates to different TCP flows in order to improve the throughput capacity of the system.

Our contributions can be summarized as follows:

1. We explore the cross-layer optimization of MAC and physical layer parameters with respect to TCP

4. Such an approach falls in the realm of RF cross-layer optimization. sending rate. At the MAC layer, we propose a scheduler that determines the rate to allocate to a TCP session from a set of channel rates. At the physical layer, we explore the set of channel rates that maximizes TCP throughput.

2. For a single TCP session, we develop an analytical expression for the steady-state throughput of a longlived TCP session in such an environment. Our model explicitly captures the dependency of the scheduler on TCP sending rate as well as the impact of the presence of two distinct rates and FERs on TCP. Each channel transmission rate results in a different roundtrip time (RTT) and FER. This system is used to study the benefits of cross-layer optimization of the dynamic rate adaptation feature of modern cellular networks with respect to TCP throughput. Indeed, a key benefit of an analytical model is the significant speedup in the numerical optimization process compared to lengthy simulations. In some instances studied in this paper, using $n s-2$ simulations to find the optimal configuration required several days of computations, while the same optimization took just a few minutes using our model.

3. We demonstrate how the analytical expression can be used to choose RF control variables that maximize TCP throughput. For example, we identify the optimal coding rates to be used in each of the two states when coding rate is used to control data rate and FER. The model is also applied to determine the optimal spreading factors, which is representative of rate control in current CDMA networks. Our studies show that throughput improvements on the order of 15 to 20 percent can be obtained for a single TCP session. Furthermore, most of benefits of adaptive rate allocation are obtained with just two channel rates.

4. Finally, we study the benefits of dynamic rate adaptation for multiple TCP sessions in a CDMA system, in particular the presence of statistical multiplexing gain. We propose two multiuser channel allocation mechanisms for such an environment: one, which relies only on coarse TCP dynamics, called $\alpha$-EMPT scheduler, and another which takes more detailed TCP rate information into consideration, called $\beta$-AWARE. The behavior of the system under these two schedulers is explored via extensive simulations over the space of input parameters $\alpha$ and $\beta$ (to be explained later). Our results indicate that multiuser channel allocation strategies have only a limited impact on TCP, and most benefits come from dynamic rate adaptation.

The remainder of this paper is structured as follows: Section 2 discusses the related work in more depth and contrasts it with our current work. In Section 3, we describe our system model and state our assumptions regarding different aspects of the system operation. The proposed channel schedulers for single-user and multiuser systems are presented in Section 4 . Section 5 presents our model for a TCP session that captures the correlation between the TCP and the scheduler. Section 6 presents our numerical results. We validate the accuracy of the model 
against $n s-2$ simulations and also explore the impact of rate adaptation on single and multi-TCP sessions through the model and simulations. Our conclusions as well as future work are discussed in Section 7.

\section{Related Work}

Numerous approaches have been proposed in the literature to optimize TCP performance in wireless networks. These approaches can be broadly categorized as either TCP enhancement approaches or link-layer optimization approaches.

The framework presented in this paper is a link-layer optimization approach that, rather than modify TCP to adapt to RF dynamics (as with TCP enhancement mechanisms), adapts the RF layer to TCP dynamics. In this view, our work is closer in philosophy to previous literature that optimizes link-layer parameters like Forward Error Correction (FEC), Automatic Repeat reQuest (ARQ), and RF scheduling to improve TCP throughput.

Barakat and Altman [4] and Liu et al. [5] analyzed the trade-off between TCP throughput and the amount of FEC added by the link layer. They showed that there exists a coding rate that maximizes TCP throughput though they only considered channel error losses. Barman et al. [6] also conducted a similar study but included the impact of signal power and ARQ as well. Baccelli et al. [7] developed an analytical model of TCP that includes the impact of congestion losses due to a finite capacity channel. They used this model to study the impact of both coding rate and processing gain on TCP throughput (see also [8], [9], and [10] for other cross-layer optimization techniques proposed to improve TCP throughput). All these previous studies, however, considered a static scenario with only a single coding rate.

Adaptive coding on the fly has been studied in [5] and [11] via simulations. In both cases, however, the scheduler behavior is agnostic to instantaneous TCP state and is based on expressions for the long-term TCP throughput.

Altman et al. [12] and Chan and Ramjee [13] modeled TCP in the presence of variable RTTs and packet losses on wireless links. However, they assumed that the variability is independent of TCP dynamics which is at odds with the environment considered here as well as the commercial environment studied in [2]. Finally, the authors of [14] studied optimization of transmission power to maximize TCP throughput. They explicitly considered TCP dynamics in the selection of the transmission power level. However, the resulting solutions were quite complex, requiring detailed TCP state knowledge. Furthermore, our focus is also different from this work since we study the impact of rate adaptation for both single and multiple TCP sessions which was not considered in [14].

\section{System Model}

In this section, we motivate the problem and present the system model including assumptions regarding different aspects of the system operation. For ease of exposition, we shall use the CDMA2000 1xRTT [1] system as an example of a practical system suited to our proposed adaptive resource allocation mechanism, although our schemes also apply equally to other wireless systems that dynamically adapt wireless channel rates in response to user sending rates.

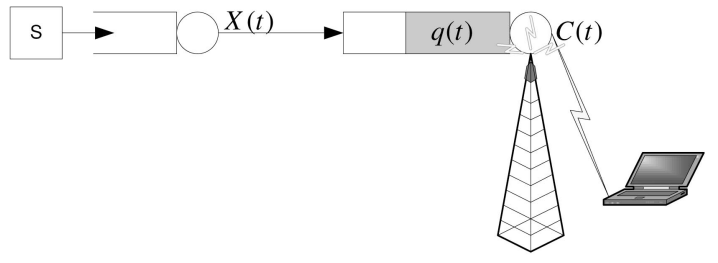

Fig. 1. Illustration of a cellular hop.

Fig. 1 depicts the wireless hop in a typical cellular network. It comprises a base station, mobile devices, and a buffer at the base station for each user. The channel scheduler resides at the base station (or in the case of CDMA2000 1xRTT, the Base Station Controller) and determines the rate allocated to each mobile session. For the purposes of this work, we focus on mobile sessions that involve TCP bulk transfers on the downlink.

The channel scheduler is assumed to have the ability to dynamically assign different wireless transmission rates (termed channels) to each user in a sector. For example, CDMA2000 1xRTT supports five different rates ranging from 9.6 to $153.6 \mathrm{Kbps}$ per user by changing processing gain and power to trade off channel error for increased capacity. The main theme of this work is to show that TCP throughput can be increased by exploiting this feature, in particular through careful selection of the channel rates and a mechanism to adaptively allocate different channel rates in response to TCP state.

Rate adaptation is also beneficial when multiple TCP sessions are in progress. Through a detailed analysis of a single TCP session in a multirate scenario, we show that the higher rate channel is not always utilized. This immediately suggests that by virtue of statistical multiplexing gain, with a few high-rate channels and careful allocation of these channels in a multisession system, TCP sessions can achieve nearly similar throughput as isolated sessions with dedicated channels. We verify and discuss this in Section 6, where it is shown that this framework and our proposed channel allocation schemes yield bandwidth/energy savings and higher throughput compared to a network with a single static channel rate.

Such a framework is also practically relevant. Again taking CDMA2000 as an example, high-rate channels are achieved by reducing processing gain (equivalently shortening the Walsh orthogonal code). This makes them much more susceptible to interference compared to low-rate channels, and consequently, only a small number of users may use a high-rate channel simultaneously. For example, 30 users may simultaneously use a $9.6-\mathrm{Kb}$ s channel but only two users may concurrently be assigned a $102.4-\mathrm{Kbps}$ channel. This is very similar to our framework wherein several TCP sessions contend for a few high-rate channels, and hence, our results are applicable to such a system.

Next, we present the system model and assumptions utilized in our work that capture the various aspects of a CDMA system mentioned above:

1. For simplicity, we focus on a two-rate system to explain our rate allocation framework. Extensions of the system model to three or more rates are straightforward. Denote by $C_{0}$ and $C_{1}$ the low-rate 
(also called fundamental) channel rate and high-rate (also called supplemental) channel rate, respectively. Clearly, $C_{0} \leq C_{1}$. At each point in time, the scheduler decides which of the two channels, the low-rate channel (rate $C_{0}$ ) or the high-rate channel (rate $C_{1}$ ), are to be assigned to a TCP session. Each active user can always be assigned a low-rate channel; however, allocation of high-rate channels is arbitrated by the channel scheduler because there are only a few highrate channels.

2. The packet error probability is implicitly assumed to be a function of the assigned rate and denoted by $p_{0}\left(p_{1}\right)$ when the assigned channel rate is $C_{0}\left(C_{1}\right)$. This is an important feature representative of current wireless systems where an increase in channel rate typically comes at the cost of increased packet error probability. For simplicity, we shall refer to $\left(p_{i}, C_{i}\right)$ together as a state or mode. We shall dwell on the relation between $p$ and $C$ in Section 6.1.

3. We assume the presence of power control to primarily combat fast fading and interference effects. This is true in current systems where fast closed-loop power control tracks a specified target SINR (or equivalently target FER).

4. We assume no (or a very small) buffer at the base station. Hence, TCP experiences congestion if its sending rate exceeds the maximum assigned channel rate.

5. Finally, in the context of multiple TCP sessions, we assume a fixed population of $N$ sessions. Each session can be assigned a low-rate channel; however, only $K \leq N$ sessions can be assigned a high-rate channel concurrently. This captures issues such as increased interference in high-rate channels and also allows us to study statistical multiplexing gain by varying $K$.

Finally, we emphasize that at this stage, no specific assumptions have been made regarding how the channel rates are achieved nor how they result in the specific channel error probabilities. Indeed, the specific relation is not required in our TCP model and only the actual variables $\left(p_{i}, C_{i}\right)$ are required. The channel rates and packet error probabilities are a function of the underlying technology that is used, e.g., adaptive modulation and spreading. This issue is addressed in detail in Section 6.

\section{4 tCP-Aware Resource Allocation}

In this section, we present our channel schedulers for single-user and multiuser systems. We first describe the single-user scheduler, and based on this, we then propose two multiuser schedulers.

\subsection{Single-User Scheduler}

The TCP-aware channel scheduler we consider resides at the base station. It is quite similar in operation to the 1xRTT scheduler described in Section 3, with the exception that it assigns a channel rate based on the user's TCP sending rate

5. This of course implies the assumption that the scheduler can measure TCP sending rate, which is defined in the next section. rather than its buffer content. Specifically, whenever the TCP sending rate exceeds (or drops below) the current channel rate, the base station increases (decreases) the channel rate (accompanied by the corresponding FER, signal power, etc.). If the session sending rate exceeds the maximum possible channel rate, the session experiences packet losses due to congestion.

As before, we utilize a two-rate system as the reference example. We note that modern cellular systems have five or more modes, i.e., they can support up to five or more different channel rates. However, obtaining succinct analytical expressions for TCP throughput even for three rates is quite difficult. Moreover, our simulations show that there is only a small improvement in system performance by considering more than two rates. We will address this issue in greater detail in Section 6.

The single-user scheduler operates as follows: The scheduler decides which of the two channel rates $C_{0}$ and $C_{1}$ are to be assigned based upon the user's TCP sending rate. If the TCP sending rate is below $C_{0}$, the scheduler assigns a channel rate of $C_{0}$, otherwise, it assigns $C_{1}$. In other words

$$
C(t)= \begin{cases}C_{0} & \text { if } X(t) \leq C_{0}, \\ C_{1} & \text { otherwise }\end{cases}
$$

where $X(t)$ is the TCP sending rate at time $t$, and $C(t)$ is the assigned channel rate.

The motivation for such a scheduler was presented in Section 1. Intuitively, when the TCP sending rate is small, a lower FER is essential (to avoid time-outs, etc.). The scheduler can exploit the knowledge of the low TCP sending rate to trade off channel rate for channel integrity. At higher TCP sending rates, it is more appropriate to assign a larger channel rate at the expense of a higher FER. This is because, even though packet loss probability due to channel errors increases, a larger channel rate prevents packet loss due to congestion, which would have happened with probability one were the rate not increased, allowing TCP to transmit at high rates for a longer time.

We note that there are several other features of TCP, for example, time-out values, window size, sending state, etc., which could potentially be utilized to improve upon our proposed scheduler. Incorporation of such features however, would make the scheduler complex to implement as well as to study. Our aim here is to propose a system that involves minimal modifications to schedulers used in current technologies like CDMA2000 1xRTT and EV-DO, and can be studied analytically. From this perspective, we believe that our proposal to incorporate knowledge of only TCP sending rate satisfies both goals.

\subsection{Multiuser Scheduler}

In this section, we propose two flow-level channel schedulers, which are extensions of the single-user scheduler described in the previous section, for channel allocation among multiple TCP sessions. We assume that all users have the same propagation delay. This is not unreasonable since all major cellular networks deploy splitTCP [15] on the downlink implying that TCP sessions on the downlink originate at a common proxy and, hence, share a common path. 
A naive extension of our single-user scheduler would be to have a dedicated high-rate channel for every user in the system. However, our analytical and simulation results for single-user system show that the high-rate channel is not fully utilized implying that such an extension would be wasteful of system resources, i.e., high-rate channels. Hence, we assume that there are only a few high-rate channels as is the case in CDMA2000 systems and propose flow-level scheduling strategies to maximize TCP throughput in a multiuser scenario.

\subsection{1 $\alpha$-EMPT Scheduler}

The $\alpha$-EMPT, which has a control parameter $\alpha$ works as follows:

1. Upon commencement, a TCP session $i$ is assigned a fundamental channel with rate $C_{0}$. Note that all $N$ sessions can be assigned fundamental channels.

2. Similar to the single-session case, the scheduler must make a decision on allocation of a high-rate channel whenever a TCP session's sending rate reaches $C_{0}$.

3. In such a scenario, if a high-rate channel is free then it is allocated to the requesting session. Otherwise, one of the $K$ users occupying the high-rate channels is preempted with probability $\alpha$ and the released channel is allocated to the requesting TCP session. Note that in this case, the preempted session faces congestion loss with probability one and halves its sending rate. Alternatively, with probability $(1-\alpha)$, the requesting session is denied the high-rate channel, in which case it is the requesting session that experiences congestion and drops its sending rate by half.

4. Whenever a user in a high-rate channel drops its sending rate (triggered by either a congestion loss or a random channel error), the user releases the highrate channel and gets assigned a fundamental channel.

We need to specify the preemption probability $\alpha$ in order to completely specify the scheduler. Furthermore, it is clear that $\alpha$, the number of supplementary channels as well as the channel rates will all impact TCP throughput. In Section 6.5, we study these various issues.

\subsection{2 $\beta$-AWARE Scheduler}

The $\beta$-AWARE scheduler is similar to the $\alpha$-EMPT scheduler, with the difference that instead of randomly selecting a user occupying the supplemental channel for preemption, it chooses a user with the largest TCP window size among those whose rate is above a factor $\beta$ of the peak capacity $C_{1}$.

The intuition behind the $\beta$-AWARE scheduler can be explained as follows: Let us assume that user $j$ has a high-rate channel and $X_{j}(t)$ is the sending rate of user $j$ at time $t\left(C_{0}<X_{j}(t)<C_{1}\right)$. We also assume that at time $t$, user $i$ requests a high-rate channel, i.e., $X_{i}(t)=C_{0}$. Consider the following scenarios:

1. If $X_{j}(t)$ is close to $C_{0}$, then there is no throughput gain in preempting user $j$ to allocate the high-rate channel to user $i$. In fact, the overall system throughput may even degrade if user $j$ is preempted.
2. If $X_{j}(t)$ is very close to $C_{1}$, then after a short period of time, user $j$ will reach $C_{1}$ and face congestion with probability one. When that happens, the high-rate channel will stay idle until another user makes a request. In this case, system throughput can be improved by preempting user $j$ to accommodate user $i$ at high rate.

$\beta$-AWARE tries to distinguish between these scenarios by predicting how much longer a high-rate user can send at high rate before reaching $C_{1}$. $\beta$-AWARE uses a threshold $\beta\left(C_{0} / C_{1} \leq \beta \leq 1\right)$ to decide when to preempt a high-rate user $j$ by considering how close $X_{j}(t)$ is to $C_{1}$. The scheduler operates similar to $\alpha$-EMPT except when contention occurs. Assume user $i$ requests a high-rate channel when all highrate channels are occupied. Let $j^{*}$ denote the user that has the highest sending rate, i.e., $j^{*}=\arg \max _{1 \leq j \leq N} X_{j}(t)$. Then, $\beta$-AWARE works as follows:

1. If $X_{j^{*}}(t) \leq \beta C_{1}$, then user $i$ 's request for a high-rate channel is denied.

2. Otherwise, user $j^{*}$ is preempted and the released high-rate channel is assigned to user $i$.

Therefore, we have a multiuser scheduler that takes into consideration the sending rate of both low-rate users and high-rate users in making scheduling decisions. Furthermore, note that $\beta=1$ corresponds to $\alpha=0$ in the $\alpha$-EMPT scheduler since in both cases this would result in no preemption. Similarly, $\beta \leq C_{0} / C_{1}$ corresponds to $\alpha=1$. In Section 6.5 , by varying the parameters $\alpha$ and $\beta$, we study a wide range of behaviors when either ignoring or incorporating TCP rate information into channel allocation decisions.

It is worth mentioning that since we consider longlasting TCP flows in a homogeneous environment where all sessions experience the same propagation delay, the above schedulers are inherently fair. All sessions are statistically treated the same by the schedulers, and hence, they achieve the same steady-state throughput.

\section{Single tCP Session Analysis}

In the previous section, we presented a TCP-aware channel scheduler for single and multiple sessions. Next, we present an analytical model for the single-session case that explicitly captures the interplay between TCP and the scheduler in a two-rate system. Such a model can be useful to quickly explore system behavior instead of utilizing lengthy simulations.

From a TCP flow perspective, such a system results in two distinct operating regimes with different channel capacities, RTTs and packet error probabilities. In this section, we present a TCP model for such a system in the single-session case that enables us to study the impact of dynamic channel allocation on TCP throughput. Extending the model to multisession case is an ongoing work. However, in Section 6, we study the scheduler performance in multiuser case through $n s-2$ simulations.

\subsection{TCP Model}

Existing TCP models typically assume that the RTT and packet loss statistics are independent of TCP dynamics in throughput calculations. However, in the wireless environment considered in this work, there exists a strong 




Fig. 2. TCP window size evolution over a variable rate channel.

correlation between the scheduler and TCP sending rate. In particular, the channel capacity $C$ that affects RTT, as well as packet loss probability $p$ are functions of the TCP sending rate. As an illustration, Fig. 2 depicts the evolution of window size of TCP in steady state when serviced by the proposed TCP-aware channel scheduler. The scheduler assigns rates based on the TCP sending rate and as can be seen, this in turn affects the window growth rates. In Section 6, we show that ignoring this dependency can result in large errors in throughput prediction.

In order to tackle the impact of the proposed channel scheduler on TCP throughput, we use the model developed by Baccelli et al. [7] for a single fixed rate channel as a starting point and develop a model that accounts for the two-rate regime. We present a more detailed explanation of our model below:

1. We assume that the TCP version is TCP Reno and model the TCP window growth in steady state as a fluid process where the window size grows linearly in the absence of loss.

2. The sender is assumed to always have data to send and, for analytical tractability, we ignore time-outs and slow start.

3. Let $W(t)$ denote the window size of TCP at time $t$ and $R(t)$ the RTT at time $t$. In the absence of a buffer, if the scheduler is in mode $i=0,1$ at time $t$, we approximate the RTT $R(t)$ as

$$
R(t)=R_{i}=a+L / C_{i},
$$

where $a$ is the round-trip propagation delay, $L$ is the packet length, and $C_{i}$ is the channel capacity in mode $i$.

4. Let $X(t)$ denote the instantaneous TCP sending rate at time $t$ in bits per second. Then, $X(t)=\frac{W(t)}{R(t)}$.

5. During congestion avoidance, the TCP window size increases by roughly one packet ( $L$ bits) every $R_{i}$ seconds in mode $i$ when there is no packet loss. We approximate this in our fluid model with a linear growth rate of $L / R_{i}$. Consequently, the sending rate grows at a linear rate of $L / R_{i}^{2} \mathrm{bits} / \mathrm{s}^{2}$ in the absence of loss. To see this, note the rate of increase of $X(t)$ is given by

$$
\frac{X\left(t+R_{i}\right)-X(t)}{R_{i}}=\frac{W\left(t+R_{i}\right)-W(t)}{R_{i}^{2}}=\frac{L}{R_{i}^{2}} .
$$

6. As in [7] and [16], we assume that the channel losses can be modeled by an inhomogeneous Poisson process with rate $p_{i} X(t), i=0,1$ at time $t$.

7. If the TCP sender is not constrained by the receiver window, then TCP experiences congestion with probability 1 when its sending rate, $X(t)$, exceeds $C_{1}$. However, if TCP is constrained by the receiver window size, the sending rate stops increasing once it reaches the receiver advertised window and it experiences only channel-related losses.

We need to approach the problem differently depending on whether the two modes (channel rates) satisfy either $C_{1} \leq 2 C_{0}$ or $C_{1}>2 C_{0}$. For the important case ${ }^{6}$ of $C_{1} \leq 2 C_{0}$, we derive expressions for mean TCP throughput for both the unconstrained rate case as well as the constrained rate case. However, we present results only for the unconstrained rate case in Section 5.2. Interested readers are referred to [17] for the analysis of the rate-constrained case. We have also obtained expressions for the TCP throughput for the case when $C_{1}>2 C_{0}$ but omit them here due to lack of space as well as the fact that most performance improvements are obtained in the region $C_{1} \leq 2 C_{0}$.

For ease of exposition, we define some notation:

1. Let $f_{i}(x, t)$ denote the density function of rate $X(t)$ at time $t$ in mode $i$. Then,

$$
f_{i}(x, t) \Delta x=\mathbb{P}\left\{x \leq X(t) \leq x+\Delta x, C(t)=C_{i}\right\},
$$

where $C(t)$ is the instantaneous channel rate at time $t$. It is clear from the above definition that

$$
f(x, t)=f_{0}(x, t)+f_{1}(x, t),
$$

where $f(x, t)$ denotes the density function of $X(t)$.

2. We also define the terms

$$
\delta_{i}=\frac{L}{R_{i}^{2}}, \quad \gamma_{i}=\frac{p_{i}}{L}, \quad \text { for } i=0,1
$$

\subsection{Throughput Analysis}

Before proceeding with the analysis, it is worthwhile to discuss an important aspect of the variable rate environment that directly affects the analysis, namely the behavior of the TCP sending rate at the channel transition points. Suppose at time $t^{-}, X\left(t^{-}\right)=C_{0}$. The corresponding window size is given by $W\left(t^{-}\right)=C_{0} R_{0}$. As per the proposed policy, the scheduler would then assign a rate of $C_{1}$ to the TCP session at time $t^{+}$resulting in a new RTT of $R_{1}$. Since TCP is a window-based protocol, the window size will be continuous at the rate transition point. Specifically, we have $W\left(t^{+}\right)=W\left(t^{-}\right)=C_{0} R_{0}$. Consequently, the new sending rate is

$$
X\left(t^{+}\right)=\frac{W\left(t^{+}\right)}{R_{1}}=\frac{R_{0} \cdot C_{0}}{R_{1}}=\frac{R_{0}}{R_{1}} X\left(t^{-}\right) .
$$

In other words, the TCP sending rate experiences a discontinuous jump by a factor of $g=R_{0} / R_{1}$ when the channel capacity transitions from $C_{0}$ to $C_{1}$. Similar arguments can be used to show that if $X(t) \geq C_{0}$ and TCP 


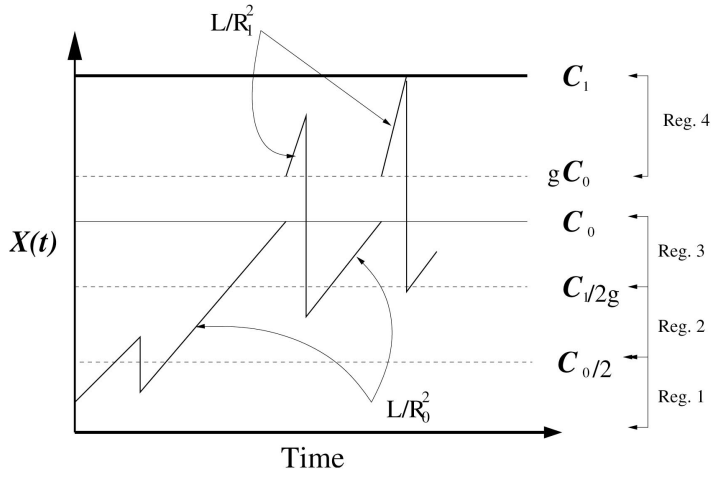

Fig. 3. Regimes for $C_{1} \leq 2 C_{0}$.

experiences a loss, the sending rate drops by a factor $1 / 2 g$. This aspect of the TCP sending rate must be accounted for in the analysis.

For purposes of analysis, we partition the range of the sending rate $X(t)$ into four different regions as shown in Fig. 3. The discontinuity in $X(t)$ when the channel rate transitions from $C_{0}$ to $C_{1}$ is clearly visible in the figure. An interesting observation is that because of the discontinuity, the sample path of $X(t)$ never resides in the region $\left(C_{0}, g C_{0}\right)$. This can be contrasted with the window size evolution in Fig. 2 which has no discontinuities.

We use techniques from fluid analysis to develop forward differential equations for the probability density function $f_{i}(x, t)$. Under the assumption that $X(t)$ has a steady-state distribution in the limit $t \rightarrow \infty$, we obtain the following system of differential equations for the distribution $f_{i}(x)$, where we denote $\frac{d}{d x} f_{i}(x)$ by $\dot{f}_{i}(x)$ :

1. $0<x<C_{0} / 2$

$$
\begin{aligned}
\delta_{0} \dot{f}_{0}(x) & =-\gamma_{0} x f_{0}(x)+4 \gamma_{0} x f_{0}(2 x), \\
f_{1}(x) & =0 .
\end{aligned}
$$

2. $C_{0} / 2<x<C_{1} / 2 g$

$$
\begin{aligned}
\delta_{0} \dot{f}_{0}(x) & =-\gamma_{0} x f_{0}(x)+4 g^{2} \gamma_{1} x f_{1}(2 g x), \\
f_{1}(x) & =0 .
\end{aligned}
$$

3. $C_{1} / 2 g<x<C_{0}$

$$
\begin{aligned}
\delta_{0} \dot{f}_{0}(x) & =-\gamma_{0} x f_{0}(x), \\
f_{1}(x) & =0 .
\end{aligned}
$$

4. $g C_{0}<x<C_{1}$

$$
\begin{aligned}
f_{0}(x) & =0, \\
\delta_{1} \dot{f}_{1}(x) & =-\gamma_{1} x f_{1}(x) .
\end{aligned}
$$

The above differential equations can be solved numerically in order to obtain the rate distribution $\left\{f_{i}(t)\right\}$. However, the actual quantity of interest for optimization is usually the mean TCP throughput, and fortunately, an analytical expression for it can be obtained, using Mellin transforms [18]. Define $\phi_{i j}$ as follows:

$$
\phi_{i j}=\frac{\gamma_{i}}{\delta_{j}}=\frac{p_{i} R_{j}^{2}}{L^{2}} .
$$

Furthermore, define

$$
\Delta(u)=\int_{C_{0}}^{C_{1}} e^{-\phi_{11} x^{2}} x^{u} d u,
$$

which is the incomplete Gamma integral, and let

$$
\Pi_{k}(u)=\frac{1}{u+2 k} \prod_{i=0}^{k-1}\left(\frac{1-2^{-u-2 i}}{u+2 i}\right) .
$$

After some algebraic manipulation (complete details are available in [19]), we obtain the final expression for TCP throughput as

$$
\bar{X}=\frac{\Delta(2)+\sum_{k \geq 0}\left(\phi_{00}\right)^{k} \Pi_{k}(2) \psi(2+2 k)}{\Delta(1)+\sum_{k \geq 0}\left(\phi_{00}\right)^{k} \Pi_{k}(1) \psi(1+2 k)} .
$$

We note that though the above analytical expression may not yield itself to direct interpretation, nevertheless, it is still quite useful since utilizing (6) to compute TCP throughput significantly speeds up computation time as opposed to simulations or numerical evaluation of the differential equations. Moreover, one can obtain simpler expressions for special cases, e.g., $p_{0}=0$, if it is desired.

\section{Numerical Results}

This section is devoted to quantifying the throughput gain obtainable with TCP-aware rate adaptation, verifying the accuracy of the analytical model as well as exploring the impact of the two resource contention mechanisms on multiple TCP sessions in a CDMA system.

Before evaluating the performance of the rate adaptation scheme, we must address the issue of how rate adaptation by the channel scheduler affects both channel capacity and packet error probability. Note that our framework does not assume any specific dependencies between the various channel rates and packet error probabilities, rather it assumes these are predetermined input variables.

As mentioned in Section 1, rate adaptation in CDMA systems can be achieved by changing any of three variables: error coding rate, the spreading factor, or modulation scheme. In this work, we study the impact of the first two variables, though our framework is equally applicable to any other means of controlling the data rate. We initially assume that the channel rate and packet error are controlled solely by changing the coding rate. This is primarily to provide a simple basis to evaluate the accuracy of the model as well as showcase its utility in a single-user framework. Section 6.1 presents the relationship we use to quantify the impact of coding rate on channel capacity and packet error probability. We use this relationship to 1 ) evaluate the accuracy of our model in Section 6.2 and 2) choose coding rates to maximize TCP throughput in Section 6.3.

In Section 6.4, we consider scenarios where resource allocation is controlled by changing the spreading factor. This 
is representative of current CDMA networks where spreading factor is the dominant control knob to adapt data rates. We evaluate the gain in TCP throughput and resultant trade-off with energy consumption as a function of the spreading factor. Finally, using the same scenario in Section 6.5, we evaluate a system with multiple TCP sessions, in particular the impact of $\alpha, \beta$, number of channels, etc., on TCP throughput.

In passing, we note that another potential control factor in wireless networks is the retransmission mechanism typically deployed at radio link layer to mitigate high FERs by retransmitting erroneous frames. Our model can easily incorporate the impact of link-layer retransmissions as a trade-off between channel rate and packet error probability similar to the coding rate studied here, though, it cannot account for the latency introduced by link-layer retransmissions. However, extensive measurements conducted over a commercial 1xRTT network by Mattar et al. [2] found that the impact of rate changes of the scheduler on TCP sending rate is far more dominant, while there is little or no correlation between TCP RTTs and the link-layer retransmissions primarily due to large RTTs and very fast retransmissions. Hence, we do not study the impact of link-layer retransmissions in this work.

\subsection{Packet Error Probability: The Variable Coding Case}

As mentioned previously, we assume that the capacity $C_{i}$ and packet error probability $p_{i}$ in mode $i$ are functions of the coding rate $\rho_{i}$. Hence, for a given bit error probability, the TCP throughput is a function of the two coding rates, i.e., $\bar{X}\left(\rho_{0}, \rho_{1}\right)$. The relation between the capacity and coding rate is straightforward and is given by $C_{i}=\rho_{i} \cdot C^{*}$, where $C^{*}$ is the uncoded channel capacity.

The packet error probability, however, is strongly dependent on not just the coding rate but also the coding scheme used. Consequently, one must either choose a specific coding scheme, or resort to bounds on the achievable packet error probability for a given coding rate. One such bound is the Gilbert-Varshamov [20] bound. This was used in [7] and we also use it as an approximation. The Gilbert-Varshamov bound is a bound on the parameters of a code of length $B$ and information bit length $K$. It specifies that there exists a minimum Hamming distance $d$ between any two codewords that must satisfy

$$
2^{B} \leq 2^{k} \sum_{j=0}^{d-1}\left(\begin{array}{l}
B \\
j
\end{array}\right),
$$

where $d-1 \leq B / 2$. Such a code can correct at most $t=$ $\lfloor(d-1) / 2\rfloor$ errors. Hence, the above relation bounds the maximum number of correctable errors for any coding rate.

Let $p_{e}$ denote the bit error probability for the wireless channel in consideration. Suppose that TCP packets have fixed size of $L$ bits. The TCP packets are broken up into radio blocks of size $B$ bits for transmission over the wireless channel. Each radio block is assumed to have $K$ bits of information and $(B-K)$ bits for coding. Hence, the coding rate is $\rho=K / B$. The packet error probability is

$$
p=1-\left(1-p_{b}\right)^{\left\lceil\frac{L}{\rho B}\right\rceil},
$$

where $p_{b}$ is the radio block error probability. Using the Gilbert-Varshamov bound, for a given coding rate, we can determine the maximum number of error bits $t$ that are correctable using the coding scheme with rate $\rho$. Then,

$$
p_{b}=\sum_{j=t+1}^{B}\left(\begin{array}{c}
B \\
j
\end{array}\right) p_{e}^{j}\left(1-p_{e}\right)^{B-j} .
$$

\subsection{Adaptive Coding Evaluation}

We begin our evaluation of the TCP model by comparing its accuracy against simulations of the TCP-aware channel scheduler implemented in $n s-2$. To be concrete in this section, we set the raw channel rate $C^{*}$ to $128 \mathrm{Kbps}$ and the two-way propagation delay $a$ to $200 \mathrm{~ms}$. TCP packet size is set to 1,024 bits. A TCP packet is divided into radio blocks of size 256 bits for transmission over the wireless channel.

The same parameters were also used for the $n s-2$ simulation. TCP Reno was chosen (since the model fits that version best) and packet errors were assumed to be independent and identically distributed. ${ }^{7}$ Since we do not account for time-outs in our model, we only simulated scenarios with large window size that result in few time-outs. Indeed, a previous study [3] has recommended the use of large window sizes in cellular networks to precisely avoid such time-outs due to bandwidth oscillations. The duration of each simulation run was $300 \mathrm{~s}$. The bit error probability was held constant for the duration of the simulation, which is true under perfect power control. Hence, the packet error probability is solely a function of the coding rate. We ran 20 simulations with different random seeds for each data point and the results reported are in the 95 percent confidence interval.

It is worth mentioning that we limited ourselves to low packet error probabilities in all the scenarios. The reasons for this are twofold. First, TCP is known to perform well only for low packet errors (less than 5 percent), and hence, it represents the region of interest. The second reason has to do with modeling the packet loss process as an inhomogeneous Poisson process which is reasonable only for low packet error probabilities. In all the scenarios considered in this paper, the model matches the simulation results closely with errors typically less than 5 percent (please see [17] for error plots).

To demonstrate the importance of capturing the correlation between TCP and the scheduler as well as the presence of two states, we plot in Fig. 4 the difference between the two-rate model (which we have shown above to be accurate) and a single-rate model that takes only one set of parameters due to coding rate $\rho_{0}$ into consideration (please see [17] for the result of considering $\rho_{1}$ ). We observe that there exist regions where there is substantial difference in predicted throughput (20 to 30 percent). Hence, a singlerate model may not be always feasible to tune performance in such systems.

\subsection{Optimal Coding Rates}

We next turn our attention to determining the coding rates that maximize TCP throughput.

7. Typically in wireless channels, errors are bursty affecting multiple packets. From the perspective of our model, this simply shows up as a single loss event. 
TABLE 2

Two-Rate Scheduler: Gain of Adaptive Coding

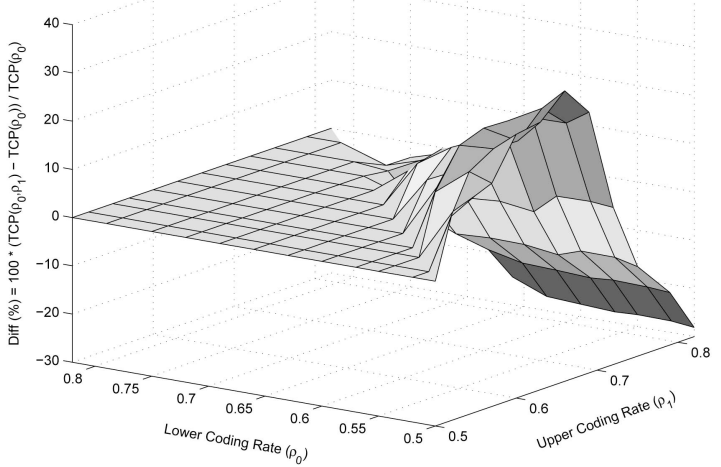

Fig. 4. Comparison between two-rate and single-rate models with parameters $\left(C_{0}, p_{0}, R_{0}\right)$ for bit error probability $\left(p_{e}\right)=10^{-2}$.

\subsubsection{Two-Rate Scheduler}

If we assume the adaptive coding case, channel rates are controlled through the coding rate. Therefore, the scheduler is essentially switching between two coding rates $\rho_{0}$ and $\rho_{1}$.

Clearly, the particular choice of $\rho_{0}$ and $\rho_{1}$ affects the achieved throughput. Intuitively, if packet error probability is close to 0 , then increasing the coding rate increases throughput as well. The reason is that, in this situation, channel rate increases as a linear function of the coding rate, whereas the packet error probability remains negligible resulting in increased throughput. However, if the packet error probability is large, then increasing the coding rate decreases throughput and eventually reduces it to 0 . Consequently, one expects the existence of a coding rate that maximizes TCP throughput. Such behavior has been previously observed for the single-rate case in [7] for finite capacity and [4] and [5] for infinite capacity models.

We extend these previous results by identifying the optimal pair of coding rates within the framework of an adaptive TCP-aware channel scheduler. Table 1 presents the pair of optimal coding rates $\tilde{\rho}_{0}$ and $\tilde{\rho}_{1}$ obtained from both the model and simulations that maximize the TCP throughput $\bar{X}\left(\rho_{o}, \rho_{1}\right)$ and the corresponding throughput for different target bit error probabilities. Observe that in most cases, there is a close match between the optimal coding rates (and corresponding throughput) obtained from the model and those from simulations. In order to quantify the benefits of using two coding rates, Table 2 presents the relative increase in throughput as a consequence of using adaptive coding compared to a single

TABLE 1

Two-Rate Scheduler: Optimal Adaptive Coding Rates $\left(C^{*}=128 \mathrm{Kbps}\right)$

\begin{tabular}{|c|cc|cc|}
\hline \multirow{2}{*}{$p_{e}$} & \multicolumn{2}{|c|}{ Analysis } & \multicolumn{2}{c|}{ Simulation } \\
\cline { 2 - 5 } & $\left(\tilde{\rho}_{0}, \tilde{\rho}_{1}\right)$ & Thr. (Kbps) & $\left(\tilde{\rho}_{0}, \tilde{\rho}_{1}\right)$ & Thr.(Kbps) \\
\hline $10^{-1}$ & $0.125,0.125$ & 10.85 & $0.0625,0.125$ & 9.22 \\
$10^{-2}$ & $0.630,0.720$ & 64.87 & $0.600,0.690$ & 62.903 \\
$10^{-3}$ & $0.820,0.910$ & 84.90 & $0.820,0.910$ & 81.43 \\
$10^{-4}$ & $0.910,0.960$ & 91.99 & $0.910,0.960$ & 91.75 \\
$10^{-5}$ & $0.960,0.990$ & 94.65 & $0.910,0.960$ & 92.43 \\
\hline
\end{tabular}

\begin{tabular}{|c|c|c|}
\hline$p_{e}$ & Analysis & Simulation \\
\hline $10^{-1}$ & $0 \%$ & $3.8 \%$ \\
$10^{-2}$ & $6.9 \%$ & $6.3 \%$ \\
$10^{-3}$ & $4.2 \%$ & $3.9 \%$ \\
$10^{-4}$ & $5.3 \%$ & $7.15 \%$ \\
$10^{-5}$ & $2.7 \%$ & $0 \%$ \\
\hline
\end{tabular}

coding rate for each bit error probability. The gain factor is defined as

$$
\text { gain }=100 \times \frac{\bar{X}\left(\tilde{\rho}_{0}, \tilde{\rho}_{1}\right)-\bar{X}(\tilde{\rho})}{\bar{X}(\tilde{\rho})},
$$

where $\tilde{\rho}$ represents the optimal coding rate for the single-rate case. Hence, for each bit error probability, the gain factor was computed by comparing throughput at the optimal coding rates for adaptive and static coding, respectively. In all the cases, we see that the optimal throughput with adaptive coding is at least equal to that of static coding and higher in several situations, with improvements of up to 7 percent.

Intuitively, the reason adaptive coding yields a gain in throughput over static coding even with the same target bit error probability is that the channel scheduler with adaptive coding exploits knowledge of TCP sending rate. Specifically, when TCP has a small window, it sends at a low rate. Hence, the channel scheduler can offer a smaller rate to the source but with a lower error probability. As the rate increases, the scheduler switches to a higher rate to cope with TCP. This is not possible in the single-rate case.

\subsubsection{Three-Rate Scheduler}

Next, we look at how much additional throughput gain is possible when the wireless scheduler can transition between three channel rates. In our current evaluation framework, this essentially means that the scheduler is switching between three coding rates $\rho_{0}, \rho_{1}$, and $\rho_{2}$. The three-rate TCP-aware scheduler with channel rates $\left(C_{0}, C_{1}, C_{2}\right)$ that correspond to coding rates $\left(\rho_{0}, \rho_{1}, \rho_{2}\right)$ operates as follows:

$$
C(t)= \begin{cases}C_{0}, & \text { if } X(t) \leq C_{0}, \\ C_{1}, & \text { if } C_{0}<X(t) \leq C_{1}, \\ C_{2}, & \text { otherwise, }\end{cases}
$$

where $C(t)$ and $X(t)$ denote the channel rate allocated to the TCP session and TCP sending rate at time $t$, respectively.

It is worth mentioning that the $n s-2$ simulations to identify the optimal rate configuration of the adaptive coding scheme is considerably time consuming even for the simpler two-rate scheduler. Computing TCP throughput via $n s-2$ for each combination of rates takes almost a day for 20 runs of simulations. Therefore, we have only simulated a three-rate scheduler in this paper. However, we expect to see similar results for channel schedulers with more than three channel rates.

Table 3 summarizes simulation results for three-rate scheduler for different BERs. In the table, $\left(\tilde{\rho}_{0}, \tilde{\rho}_{1}, \tilde{\rho}_{2}\right)$ denotes the optimal coding rates corresponding to three channel rates used by the scheduler that maximize TCP throughput. 
TABLE 3

Three-Rate Scheduler: Optimal Adaptive Coding Rates $\left(C^{*}=128 \mathrm{Kbps}\right)$

\begin{tabular}{|c|c|c|c|}
\hline$p_{e}$ & $\left(\tilde{\rho}_{0}, \tilde{\rho}_{1}, \tilde{\rho}_{2}\right)$ & Thr. (Kbps) & Gain \\
\hline $10^{-1}$ & $0.03125,0.06250,0.125$ & 8.81 & $0 \%$ \\
$10^{-2}$ & $0.600,0.690,0.720$ & 64.25 & $8.5 \%$ \\
$10^{-3}$ & $0.790,0.870,0.910$ & 82.54 & $5.33 \%$ \\
$10^{-4}$ & $0.870,0.910,0.960$ & 91.67 & $7.06 \%$ \\
$10^{-5}$ & $0.910,0.960,0.960$ & 92.43 & $0 \%$ \\
\hline
\end{tabular}

We have also computed the gain factor representing the percentage of throughput improvement compared to static coding throughput as defined before.

An interesting observation is that, for all simulated cases, the throughput gain of three-rate scheduler is roughly equal to that of the two-rate scheduler considering the margin of simulation error. In other words, most of the throughput improvement is already achieved by using only two rates and increasing the number of channel rates does not further improve the throughput.

\subsection{Optimal Processing Gain}

In the previous sections, we assumed that the trade-off between the channel capacity and FER was controlled by the coding rate. Next, we study scenarios that are more representative of current CDMA cellular networks. In CDMA networks, the dominant RF control variable that governs this trade-off is the spreading factor. The spreading factor is defined as the ratio of the CDMA chip rate to the actual data rate and relates the channel capacity and the bit error probability in the following manner. Let $W$ denote the chip rate of the CDMA system and $r_{i}$ the spreading factor allocated in mode $i$. Then, the data rate $C_{i}$ and Bit Energy to Noise Ratio are given by

$$
C_{i}=\frac{W}{r_{i}}, \quad \frac{E_{b}^{i}}{I_{0}}=\frac{r_{i} \cdot E_{c}}{I_{0}},
$$

where $E_{c}$ is the chip energy of the data signal, and $I_{0}$ is the wide-spectrum interference. It should be clear from the above relations that as we decrease $r_{i}$, the channel capacity $C_{i}$ increases, but the SINR, which directly affects the bit error probability, decreases.

Typically, the bit error probability is extremely sensitive to SINR and hence, in practice, some amount of power control is required to prevent a steep rise in the error probability when the spreading factor is decreased. Specifically, the chip energy is boosted by a small factor that is a function of the spreading rate to prevent a sharp drop in the quality of service. Consequently, the relationship between the SINR and the spreading factor $r_{i}$ is modified slightly to

$$
\frac{E_{b}^{i}}{N_{0}}=\frac{E_{c} \cdot r_{i} E\left(r_{i}\right)}{I_{0}},
$$

where $E\left(r_{i}\right)$ is a decreasing function of $r_{i}$ and represents the factor by which the original chip energy $E_{c}$ is boosted. Hence, in addition to the spreading factor, one can also control the bit error probability by an appropriate choice of the function $E(r)$, which we shall denote as the energy profile.

We now demonstrate how our model can be used to study the impact of rate adaptation in such an environment. Since signal power is also a resource in the above framework, we must look at both the mean TCP throughput as well as energy consumption. To quantify the latter, we use the notion of normalized energy consumption. It represents the average energy spent per bit as a function of TCP throughput and is computed as follows: The energy spent per bit in mode $i$ is given by $E_{b}^{i}=E_{c} \cdot\left(r_{i} E\left(r_{i}\right)\right)$. Further, assume that the mobile session spends a fraction of time $\pi_{i}$ in mode $i$ and achieves an average throughput $\bar{X}_{i}$ in that mode. Then, the normalized energy consumption is given by

$$
\begin{aligned}
E_{\text {norm }} & =\frac{1}{\bar{X}} \sum_{i} \pi_{i} \bar{X}_{i} \cdot E_{b}^{i}=\frac{1}{\bar{X}} \sum_{i} \widehat{f}_{i}(2) E_{b}^{i} \\
& =E_{c} \frac{1}{\bar{X}} \cdot \sum_{i} \widehat{f}_{i}(2) r_{i} E\left(r_{i}\right) .
\end{aligned}
$$

In our experiments, we set the chip rate $W$ to 1.2288 Mchips/s and the basic pilot RSSI $\left(E_{c} / I_{0}\right)$ to $-7 \mathrm{~dB}$ based on CDMA2000 standards. We assume QPSK modulation (also a CDMA2000 standard) and a fixed coding rate of 0.6. The spreading factor $r_{i}$ was allowed to take values from the set $\{4,12,16\}$, which gives a rate set of $\{76.8,102.4$, 153.6 $\mathrm{Kbps}$. We note that 78.6 and $153.6 \mathrm{Kbps}$ are the two highest data rates available in CDMA2000 1xRTT with Radio Configuration Type 3 [1]. We tested different energy profiles $E(r)$ and present results for two of them, denoted by $E_{1}$ and $E_{2}$.

\subsubsection{Two-Rate Scheduler}

Table 4 presents the throughputs obtained with different rate combinations for the two-rate case under energy profile $E_{1}$ (we omit detailed $E_{2}$ results due to space constraints.). The first column in each table represents the channel rates (and hence, spreading factors) used. The last column presents the energy consumption for each configuration, measured in multiples of $E_{c}$. The optimal configuration of spreading factors, which was chosen based on the maximum throughput, is marked in bold for both the

TABLE 4

Two-Rate Scheduler: Spreading Factors $\left(C^{*}=153.6 \mathrm{Kbps}\right)$ Energy Profile $E_{1}$

\begin{tabular}{|c|c|c|c|c|}
\hline \multirow{2}{*}{$\left(C_{0}, C_{1}\right)$ Kbps } & \multicolumn{2}{|c|}{ Analysis } & \multicolumn{2}{c|}{ Simulation } \\
\cline { 2 - 5 } & Thr. (Kbps) & $\begin{array}{c}\text { Energy } \\
\text { Consumption }\left(\cdot E_{c} \mathrm{~J} / \mathrm{bit}\right)\end{array}$ & Thr. (Kbps) & $\begin{array}{c}\text { Energy } \\
\text { Consumption }\left(\cdot E_{c} \mathrm{~J} / \mathrm{bit}\right)\end{array}$ \\
\hline $76.8,102.4$ & 74.545 & 13.77 & 74.350 & 13.77 \\
$76.8,153.6$ & 89.83 & 13.08 & 86.793 & 13.12 \\
$\mathbf{1 0 2 . 4}, \mathbf{1 5 3 . 6}$ & $\mathbf{9 8 . 1 8 0}$ & $\mathbf{1 3 . 2 6}$ & $\mathbf{9 5 . 3 1 6}$ & $\mathbf{1 3 . 2 8 4}$ \\
\hline
\end{tabular}


TABLE 5

Two-Rate Scheduler: Max Rate Configuration

\begin{tabular}{|c|cc|cc|}
\hline \multirow{2}{*}{$\begin{array}{c}\text { Energy } \\
\text { Profile }\end{array}$} & \multicolumn{2}{|c|}{ Analysis } & \multicolumn{2}{c|}{ Simulation } \\
\cline { 2 - 5 } & Rate Gain & Eng. Sav. & Rate Gain & Eng. Sav. \\
\hline$E_{1}$ & $10.8 \%$ & $-3.5 \%$ & $14.95 \%$ & $-3.5 \%$ \\
$E_{2}$ & $15.8 \%$ & $-4.8 \%$ & $20.5 \%$ & $4.2 \%$ \\
\hline
\end{tabular}

single- and the two-rate cases. The gain in TCP throughput and difference in energy consumption between the adaptive and static cases, under their respective optimal configurations, are shown in Table 5 for both energy profiles. The energy savings is computed as

$$
\text { Energy Savings }=100 \times \frac{E_{\text {norm }}^{\text {static }}-E_{\text {norm }}^{\text {adaptive }}}{E_{\text {norm }}^{\text {static }}} .
$$

There are a number of interesting observations regarding the results. From Table 5, the analytical model indicates that a small increase in energy consumption, on the order of 3 to 4 percent, in conjunction with a two-rate strategy boosts TCP throughput by 10 to 15 percent as compared to the single-rate scenario even though the allowed peak channel capacities are the same. The rate gain and energy saving predictions of the simulation are comparable to those of the model for $E_{1}$ and $E_{2}$. Interestingly, simulation predicts higher benefits than the model for energy savings in the case of $E_{2}$. In fact, it predicts a positive energy savings of 4.2 percent.

The differences in energy prediction for $E_{2}$ are due to the model and simulation choosing different optimal configurations in the single-rate case, while yielding the same configuration in the two-rate case. Specifically, the model overestimated (by about 9 percent) the TCP throughput in the single-rate case for the highest rate configuration of 153.6 Kbps, causing it to pick that as the optimal. In comparison, the simulation chose the $102.4-\mathrm{Kbps}$ rate which has higher normalized energy consumption.

\subsubsection{Three-Rate Scheduler}

We have also simulated a three-rate scheduler that utilizes all three rates $\{76.8,102.4,153.6\} \mathrm{Kbps}$ and the same energy profiles used in the previous section. Table 6 summarizes the results for throughput gain using the three-rate scheduler. Comparing the throughput and energy gains for the two-rate system in Table 5, we see that they are almost the same, indicating that two channel rates yield a large fraction of the benefits.

\subsection{Multiuser Scheduler Performance}

In previous sections, we showed that by exploiting dynamic rate adaptation, it is possible to improve system throughput by 10 percent to 20 percent for a single TCP session. We next explore the impact of rate adaptation when multiple TCP sessions are present by studying the performance of the two proposed high-rate channel allocation algorithms: $\alpha$-EMPT and $\beta$-AWARE.

\subsection{1 $\alpha$-EMPT Performance Evaluation}

Our first set of results pertain to the simpler $\alpha$-EMPT scheduler (a brief preliminary analysis appeared in [21]). For
TABLE 6

Three-Rate Scheduler: Spreading Factors $\left(C^{*}=153.6 \mathrm{Kbps}\right)$

\begin{tabular}{|c|c|c|c|}
\hline Energy Profile & Max Thr. (Kbps) & Thr. Gain & Energy Sav. \\
\hline$E_{1}$ & 95.209 & $14.8 \%$ & $-3.8 \%$ \\
$E_{2}$ & 93.147 & $20.03 \%$ & $4.2 \%$ \\
\hline
\end{tabular}

purposes of our evaluation, we utilize the same scenario as in Section 6.4 for a CDMA system. Specifically, we assume that rate adaptation is carried out by varying processing gain. Channel error probabilities were determined using the energy profile $E_{1}$ to be $\{0,7.7,8.7,43.2\} \times 10^{-4}$ for channel rates of $\{9.6,76.8,102.4,53.6\} \mathrm{Kbps}$, respectively.

Fig. 5 plots the TCP throughput as a function of $\alpha$ and the number of supplemental channels $K$ for $N=10$ users. The supplemental channel rate was set to $153.6 \mathrm{Kbps}$ in Figs. 5a, 5b, and 5c, and 102.4 Kbps in Fig. 5d. The figures show the evolution of throughput for fundamental channel rates of $102.4,76.8$, and $9.6 \mathrm{Kbps}$.

These three scenarios provide several interesting observations regarding the role of $\alpha$, the channel rates $C_{0}, C_{1}$, and the number of supplemental channels $K$, which help explain the behavior of the $\alpha$-EMPT scheduler:

1. For a fixed $\alpha$, the TCP throughput is obviously an increasing function of $K$. However, the throughput gain diminishes with increasing $K$. This can be directly attributed to the statistical multiplexing gain. Application of the single-session analytical model developed in Section 5 reveals that the supplemental channel utilization is around 45 and 70 percent when the fundamental channel rates are 102.4 and $76.8 \mathrm{Kbps}$, respectively. This indicates that the high-rate channel is not continuously occupied, especially in the first case and, hence, can be shared to a certain extent among multiple sessions. Consequently, initial increments of supplemental channels (from $K=1$ ) yield almost proportional increments in throughput because it reduces contention. However, throughput saturation sets in once $K$ is large enough to satisfy the high-rate utilization requirements: the smaller the single-session high-rate utilization, the faster the saturation, and hence, fewer channels are required. For example, when the fundamental channel rate is $102.4 \mathrm{Kbps}$ (Fig. 5a), almost all throughput gain is obtained with about five supplemental channels since TCP sessions utilize it at most 45 percent of the time. We shall explore this further in Section 6.5.3.

2. For small values of $K$, the throughput is sensitive to the choice of $\alpha$ and its behavior depends on the fundamental and supplemental channel rate combination. As $K$ increases, the throughput becomes less sensitive to $\alpha$ since supplemental channels are increasingly available when requested and the contention likelihood decreases.

It is worthwhile to further explore the detailed behavior of TCP throughput as a function of $\alpha$ for small values of $K$. For ease of exposition, consider $K=1$ in a low-loss system, 


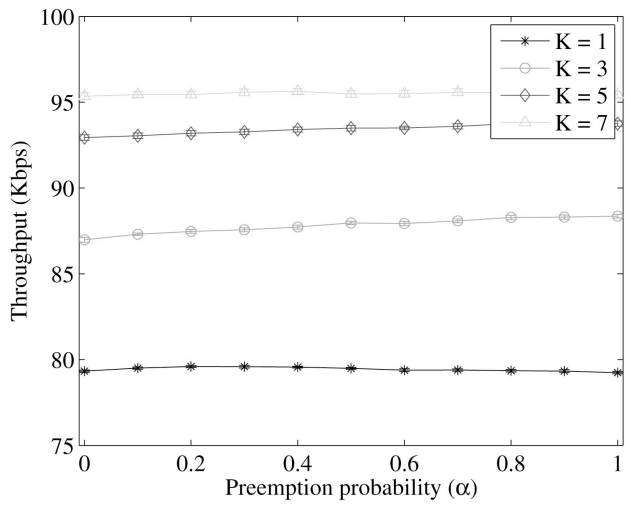

(a)

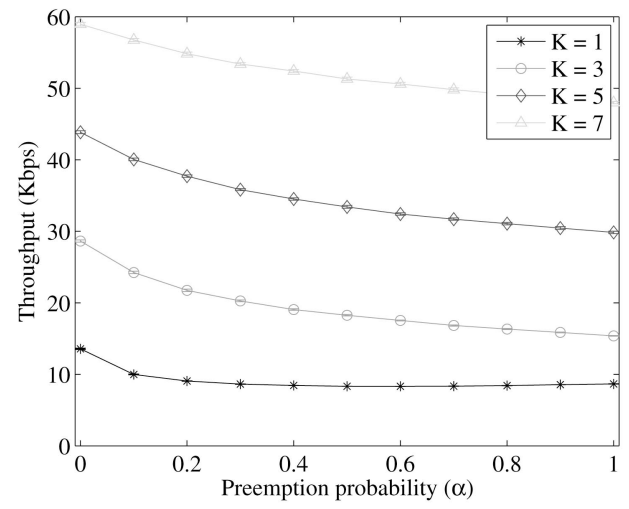

(c)

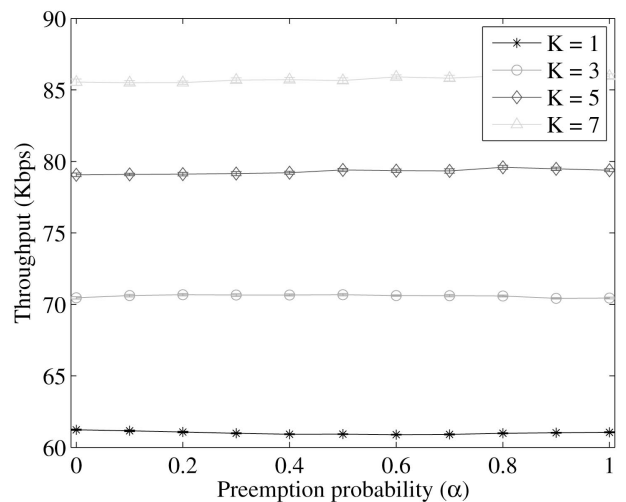

(b)

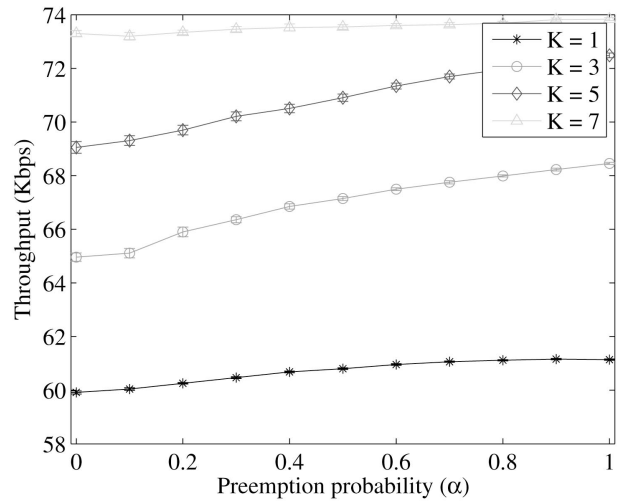

(d)

Fig. 5. Impact of $\alpha$ on TCP throughput for $N=10$. (a) $C_{0}=102.4 \mathrm{Kbps}$ and $C_{1}=153.6 \mathrm{Kbps}$. (b) $C_{0}=76.8 \mathrm{Kbps}$ and $C_{1}=153.6 \mathrm{Kbps}$. (c) $C_{0}=9.6 \mathrm{Kbps}$ and $C_{1}=153.6 \mathrm{Kbps}$. (d) $C_{0}=76.8 \mathrm{Kbps}$ and $C_{1}=102.4 \mathrm{Kbps}$.

i.e., $p_{1} \rightarrow 0 .{ }^{8}$ When $\alpha=0$, a TCP session that is granted a supplemental channel can fully utilize the high-rate channel (under the low-loss assumption) until it experiences congestion (when TCP transmission rate hits $C_{1}$ ). However, this can also lead to a lower likelihood of occupancy of the high-rate channel (i.e., it stays empty) since another session may not be available to exploit the available channel when the current session relinquishes it (see Fig. 6).

When $\alpha=1$, the supplemental channel occupancy increases (assuming sufficient requests) since the release of the high-rate channel is due to preemption. However, this can result in lower efficiency compared to $\alpha=0$ if the supplemental channel rate is large, since the session may get preempted before it can sufficiently increase its transmission rate (see Fig. 6). To summarize, the trade-off between these two factors, which in turn is determined by the relative values of $C_{0}$ and $C_{1}$, governs the TCP throughput as a function of $\alpha$.

This behavior is exemplified in Fig. 5. When $C_{0} \ll C_{1}$ (e.g., [9.6, 153.6] Kbps in Fig. 5c), the high-rate channel utilization dominates since a session in the high-rate channel can gain significant increase in throughput compared to the fundamental channel. Furthermore, since the fundamental channel is small, request rate for the high-rate channel is

8. Intuitively, as loss rate becomes high, performance becomes insensitive to $\alpha$ since a session frequently experiences channel error in the supplemental channel. large enough to avoid any potential loss of occupancy. Consequently, $\alpha=0$ is optimal because it allows a session to fully utilize the channel. As $C_{0}$ increases relative to $C_{1}$, the gain in throughput from utilization of the high-rate channel diminishes (since $C_{0}$ is already large) and request rates reduce (because sessions spend more time in $C_{0}$ as described by our model earlier). Hence, the high-rate channel occupancy factor becomes more important. Figs. $5 \mathrm{a}$ and $5 \mathrm{~d}$ illustrate this scenario. Here, $2 C_{0}>C_{1}$, and $\alpha=1$ maximizes occupancy, which yields the optimal throughput. An intermediate case is $2 C_{0}=C_{1}$ (Fig. 5b), where the two factors balance each other. This results in a TCP throughput insensitive to $\alpha$.

Next, we look at the impact of $\alpha$ on TCP throughput gain. In Fig. 7, we have plotted the maximum throughput gain of $\alpha$-EMPT across different channel configurations for

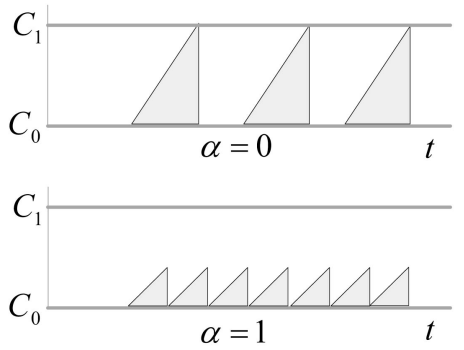

Fig. 6. Impact of $\alpha$ on supplemental channel utilization. 


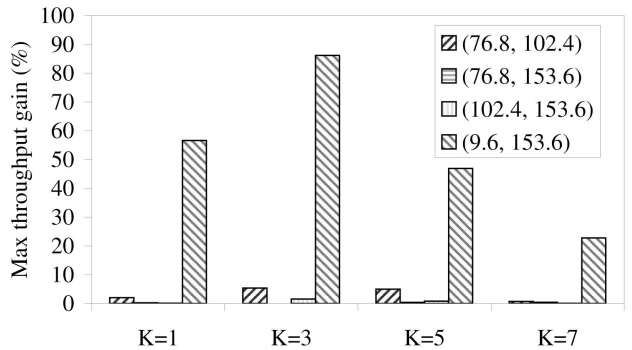

Fig. 7. Impact of $\alpha$ across all scenarios.

$N=10$ users and various supplemental channels $K$. The gain was computed (over the entire range of $\alpha$ ) as

$$
\text { Max gain }=100 \times \frac{\max _{\alpha} \bar{X}_{\alpha-\mathrm{EMPT}}-\min _{\alpha} \bar{X}_{\alpha-\mathrm{EMPT}}}{\max _{\alpha} \bar{X}_{\alpha-\mathrm{EMPT}}} .
$$

One can clearly see that the $\alpha$-EMPT scheduler, and in particular the value of $\alpha$ is influential when the gap between $C_{0}$ and $C_{1}$ is very large $\left(C_{0}=9.6\right.$ and $\left.C_{1}=153.6 \mathrm{Kbps}\right)$, resulting in throughput gains of 60 to 90 percent by appropriately tuning $\alpha$. However, when $C_{0}$ is closer to $C_{1}\left(\geq C_{1} / 2\right)$, performance is almost insensitive to $\alpha$ with small gains of about 2-5 percent.

\subsection{2 $\beta$-AWARE Performance Evaluation}

Given the relative insensitivity of TCP throughput to the $\alpha$-EMPT scheduler when the gap between $C_{0}$ and $C_{1}$ is not large, we next turn to the more sophisticated scheduler $\beta$-AWARE.

We studied the impact of the $\beta$-AWARE scheduler on TCP throughput as a function of $\beta$ and found it to have a similar dependency as the $\alpha$-EMPT scheduler on the gap between $C_{0}$ and $C_{1}$ as well as number of supplemental channels. This results are shown in Fig. 8.

Fig. 9 presents a comparison in performance between $\beta$-AWARE and $\alpha$-EMPT schedulers across different rate configurations, number of users, and supplemental channels (the numbers in brackets represent $(N, K)$ ). It shows the gain achieved in throughput with the best configuration of the $\beta$-AWARE scheduler through appropriate selection of $\beta$ when compared against the best configuration of the $\alpha$-EMPT scheduler. In other words, the gain was computed as

$$
\text { Max gain }=100 \times \frac{\max _{\beta} \bar{X}_{\beta-\text { AWARE }}-\max _{\alpha} \bar{X}_{\alpha-\mathrm{EMPT}}}{\max _{\alpha} \bar{X}_{\alpha-\mathrm{EMPT}}} .
$$

Somewhat surprisingly, we observe that in all scenarios the $\beta$-AWARE scheduler yields little benefit over the $\alpha$-EMPT scheduler, with about 2-5 percent improvement. We note that by tuning $\beta$ and $\alpha$, we cover a large range of potential behaviors. This leads us to conjecture that TCP throughput in such situations is relatively insensitive to channel allocation strategy when $C_{0}$ and $C_{1}$ do not differ by a large value and most of the gain in throughput is achieved through rate adaptation.

\subsubsection{Multiplexing Gain with Multiple Flows}

This leads us to the final aspect of the study of benefits of rate adaptation in TCP multisession systems, specifically resource efficiency. As was alluded to earlier when

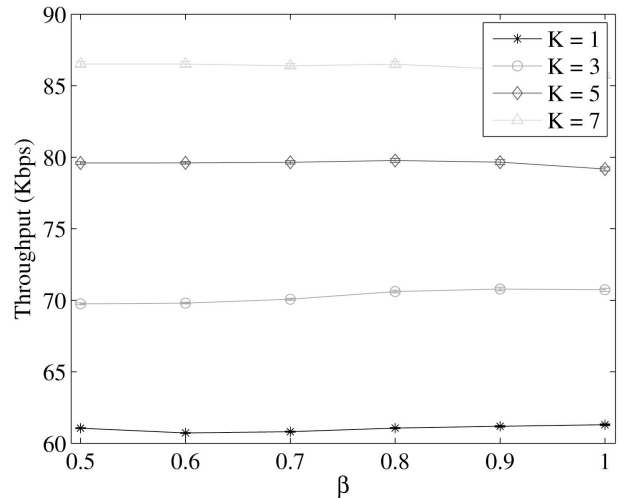

(a)

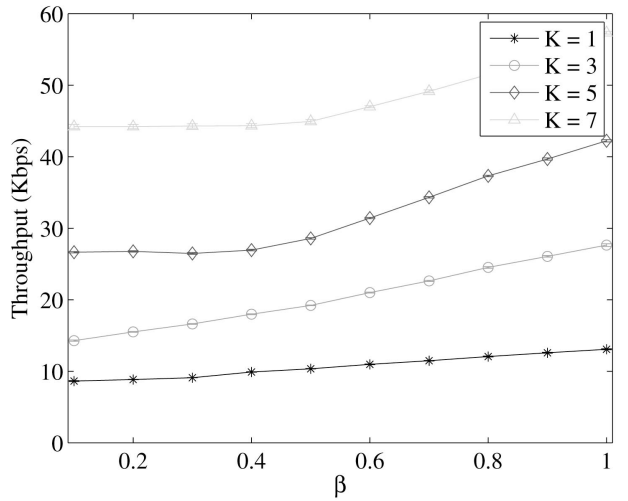

(b)

Fig. 8. Impact of $\beta$ on TCP throughput for $N=10$. (a) $C_{0}=76.8 \mathrm{Kbps}$ and $C_{1}=153.6 \mathrm{Kbps}$. (b) $C_{0}=9.6 \mathrm{Kbps}$ and $C_{1}=153.6 \mathrm{Kbps}$.

discussing the $\alpha$-EMPT scheduler, results from the singlesession analytical model indicate that in a multirate system, the high-rate channel is not fully utilized. This can lead to significant savings. In CDMA systems, high-rate channels are achieved by reducing the orthogonal Walsh code length. Since they are highly susceptible to interference, it limits the number of simultaneous high-rate channels that can be supported (the higher the rate, the fewer the channels), which in turn affects capacity. Consequently, it is desirable to provide throughput gains while at the same time efficiently utilizing high-rate channels.

By exploiting the statistical multiplexing gain inherent in rate adaptation, we demonstrate that one can achieve similar or higher throughput as with single-rate channels but with a lower total bandwidth.

Fig. 10 presents the throughput and bandwidth savings when using multirate channels in a multiuser system

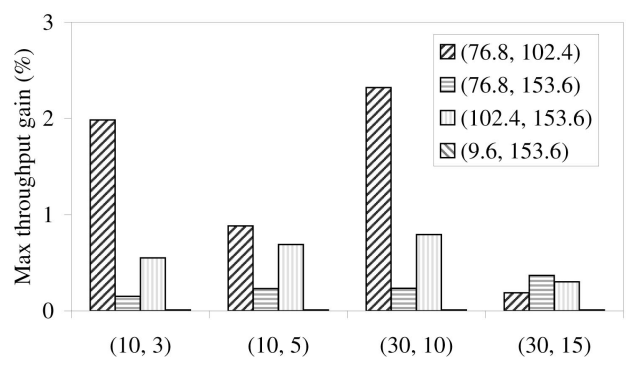

Fig. 9. Comparison of $\alpha$-EMPT versus $\beta$-AWARE scheduler. 


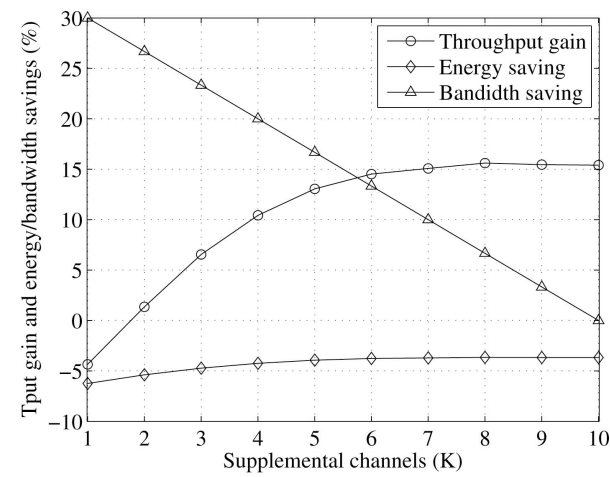

(a)

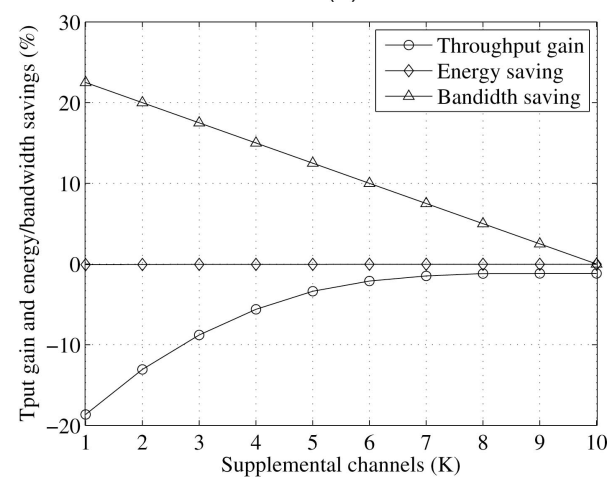

(b)

Fig. 10. Throughput gain and energy saving as function of $K$. (a) Profile $E_{1}: C=153.6,\left(C_{0}=102.4, C_{1}=153.6\right)$ Kbps. (b) Profile $E_{2}: C=102.4$, $\left(C_{0}=76.8, C_{1}=153.6\right) \mathrm{Kbps}$.

compared to single-rate channel allocation for the two different energy profiles $E_{1}$ and $E_{2}$. For purposes of comparison, the channel rate in the single-rate case was chosen to be the one that yields the maximum throughput as computed in Section 6.4. To demonstrate the impact of rate adaptation, for the multirate case, we deliberately chose rates such that the fundamental channel rate was lower than the single-rate channel allocation even if it was suboptimal in the sense that it did not yield the maximum throughput computed in Section 6.4.

The total bandwidth consumed in the single-rate case was given by $N C$, where $C$ is the single channel rate and by $(N-K) C_{0}+K C_{1}$ in the multirate case. Fig. 10 plots the percentage difference for achieved throughput and consumed bandwidth between the multirate and single-rate cases. As can be clearly seen, for energy profile $E_{1}$, the multirate system consumes less bandwidth and yields higher throughput gains of about 15 percent albeit at the cost of a small increase in energy. With energy profile $E_{2}$, when $K \geq 6$, the multirate system yields almost the same throughput as the single-rate system but consumes about 10 to 15 percent less bandwidth. The figures also clearly indicate the benefit of the multiplexing gain, in that the gains saturate around $K=5$ channels for energy profile $E_{1}$ and $K=7$ channels for $E_{2}$, i.e., performance is close to that achieved with $K=N$ channels.

\section{CONCLUSION}

In this paper, we have proposed techniques for optimizing the rate adaptation feature of modern CDMA systems in a
TCP-aware fashion. Our proposed system adapts its channel rate in response to the TCP sending rate, allowing it to trade off channel rate and FER in a TCP-friendly manner.

For the single TCP session case, we developed an analytical model for a two-state system that explicitly accounts for the interaction between our proposed scheduler and TCP dynamics as well as the presence of two distinct regimes in terms of channel rate, packet error probability, and RTT. The model was utilized to show how to select either error coding or processing gain in optimizing the rate adaptation scheme to improve TCP throughput. Improvements of 15 to 20 percent were observed compared to a system that does not exploit rate adaptation. Furthermore, we also evaluated systems that use three channel rates via simulations and observed that they yield no significant gains compared to a two-rate system.

Finally, we extended the rate adaptation framework to multiple TCP sessions in a CDMA system. In such an environment, the number of high-rate channels is less than the number of active users, and thus, the high-rate channels must be shared in a fair fashion. We proposed two channel allocation policies based on preemption and presented extensive simulation results on their performance. Our results indicated that the rate adaptation feature with selected rate combinations, rather than the channel allocation policy, is affecting the system throughput. We also showed that by exploiting statistical multiplexing gain, a system with just a few high-rate channels achieves per-session throughput close to that of a single-session system with potential savings in both bandwidth and energy budget.

\section{ACKNOWLEDGMENTS}

A preliminary version of this paper appeared in the Proceedings of ACM MobiCom '06, Los Angeles, California, USA, 24-29 September, 2006.

\section{References}

[1] Telecommunications Industry Assoc., TIA EIA IS-2000, www.tiaonline.org/standards/sfg/imt2k/cdma2000/, Mar. 2000.

[2] K. Mattar, A. Sridharan, H. Zang, I. Matta, and A. Bestavros, "TCP over CDMA2000 Networks: A Cross-Layer Measurement Study," Proc. Eight Int'l Conf. Passive and Active Network Measurement (PAM '07), Apr. 2007.

[3] E. Chaponniere, S. Kandukuri, and W. Hamdy, "Effect of Physical Layer Bandwidth Variation on TCP Performance in CDMA2000," Proc. 57th IEEE Vehicular Technology Conf. (VTC Spring '03), pp. 336-342, Apr. 2003.

[4] C. Barakat and E. Altman, "Bandwidth Tradeoff between TCP and Link-Level FEC," Computer Networks, vol. 39, no. 5, pp. 133-150, 2002.

[5] B. Liu, D.L. Goeckel, and D. Towsley, "TCP-Cognizant Adaptive Forward Error Correction in Wireless Networks," Proc. IEEE Global Telecomm. Conf. (GLOBECOM '02), Nov. 2002.

[6] D. Barman, I. Matta, E. Altman, and R.E. Azouzi, "TCP Optimization through FEC, ARQ and Transmission Power Trade Offs," Proc. Conf. Wired/Wireless Internet Comm. (WWIC '04), Feb. 2004.

[7] F. Baccelli, R. Cruz, and A. Nucci, "CDMA Channel Parameters Maximizing TCP Throughput," Proc. Workshop Information Theory and Its Applications (ITA '06), Feb. 2006.

[8] A. Chockalingam, E. Altman, J.V.K. Murthy, and R. Kumar, "Cross-Layer Design for Optimizing TCP Performance," Proc. IEEE Int'l Conf. Comm. (ICC '05), May 2005. 
[9] M. Zorzi, A. Chockalingam, and R.R. Rao, "Throughput Analysis of TCP on Channels with Memory," IEEE J. Selected Areas in Comm., vol. 18, no. 7, pp. 1289-1300, 2000.

[10] A. Chockalingam and G. Bao, "Performance of TCP/RLP Protocol Stack on Correlated Rayleigh Fading DS-CDMA Links," IEEE Trans. Vehicular Technology, vol. 49, no. 1, pp. 28-33, 2000.

[11] K.L. Gray and D.L. Noneaker, "The Effect of Adaptive-Rate Coding on TCP Performance in Wireless Communications," Proc. IEEE/AFCEA Conf. Information Systems for Enhanced Public Safety and Security (EUROCOMM), 2000.

[12] E. Altman, C. Barakat, and V.M.R. Ramos, "Analysis of AIMD Protocols over Paths with Variable Delay," Proc. IEEE INFOCOM '04, Mar. 2004.

[13] M.C. Chan and R. Ramjee, "TCP/IP Performance over 3G Wireless Links with Rate and Delay Variation," Proc. ACM MobiCom '02, pp. 71-82, 2002.

[14] J.P. Singh, Y. Li, and N. Bambos, "Channel State Awareness Based Transmission Power Adaptation for Efficient TCP Dynamics in Wireless Networks," Proc. IEEE Int'l Conf. Comm. (ICC '05), May 2005.

[15] W. Wei, C. Zhang, H. Zang, J. Kurose, and D. Towsley, "Inference and Evaluation of Split-Connection Approaches in Cellular Data Networks," Proc. Seventh Int'l Conf. Passive and Active Network Measurement (PAM '06), Mar. 2006.

[16] V. Misra, W.B. Gong, and D. Towsley, "Fluid-Based Analysis of a Network of AQM Routers Supporting TCP Flows with an Application to RED," Proc. ACM SIGCOMM '00, pp. 151-160, Aug. 2000.

[17] M. Ghaderi, A. Sridharan, H. Zang, D. Towsley, and R. Cruz, "TCP-Aware Resource Allocation in CDMA Networks," Technical Report RR06-ATL-030566, Sprint Advanced Technology Labs, Mar. 2006.

[18] F. Baccelli and K.B. Kim, "TCP Throughput Analysis Under Transmission Error and Congestion Losses," Proc. IEEE INFOCOM '04, Mar. 2004.

[19] M. Ghaderi, A. Sridharan, H. Zang, D. Towsley, and R. Cruz, "TCP-Aware Resource Allocation in CDMA Networks," Proc. ACM MobiCom'06, pp. 215-226, Sept. 2006.

[20] F.J. McWilliams and N.J.A. Sloane, The Theory of Error-Correcting Codes. North-Holland, 1977.

[21] M. Ghaderi, A. Sridharan, H. Zang, D. Towsley, and R. Cruz, "Modeling TCP in a Multi-Rate Multi-User CDMA System," Proc. Networking, May 2007.

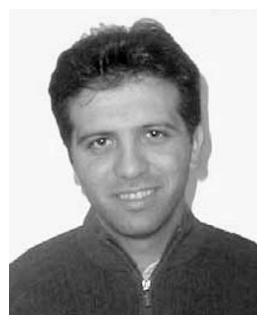

Majid Ghaderi received the BSc and MSc degrees from Sharif University of Technology and the $\mathrm{PhD}$ degree from the University of Waterloo, all in computer science. He is an assistant professor in the Department of Computer Science, University of Calgary, Alberta, Canada. Before joining the University of Calgary, he was a postdoctoral research associate in the Department of Computer Science, University of Massachusetts, Amherst. His research interests include wireless networking and mobile computing with emphasis on resource allocation, network optimization, and the modeling and performance analysis of network protocols. He is a member of the IEEE.

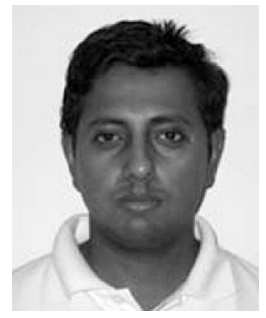

Ashwin Sridharan received the master's degree from the Indian Institute of Science, Bangalore, India in 1999 and the PhD degree from the University of Pennsylvania in 2004, where he worked on traffic engineering and routing algorithms. He has been a research scientist at Sprint, Burlingame, California, since 2004. His current interests include performance enhancement of wireless networks, fast and accurate sampling, and more generating data mining of network information. He has participated as a chair, a committee member, and a reviewer in various networking conferences. $\mathrm{He}$ is a member of the IEEE.

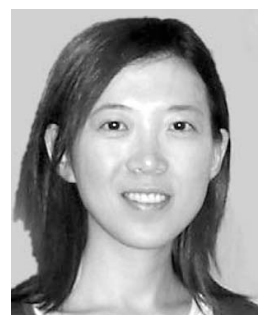

Hui Zang received the BS degree in computer science from Tsinghua University, Beijing, in 1997 and the MS and PhD degrees in computer science from the University of California, Davis, in 1998 and 2001, respectively. She is a research scientist at Sprint, Burlingame, California. Her research interests include performance and security issues of various types of networks including cellular, IP, and optical networks. She was one of the guest editors of the IEEE Network special issue on "Traffic Engineering in Optical Networks." She is the author of the book WDM Mesh Networks-Management and Survivability (Kluwer Academic, 2002). She has published more than 50 conference papers and journal articles and currently has three US patents granted and over 10 pending in the field of networking and communications. She is a senior member of the IEEE.

Don Towsley received the BA degree in physics and the $\mathrm{PhD}$ degree in computer science from the University of Texas in 1971 and 1975, respectively. He is currently a distinguished professor in the Department of Computer Science, University of Massachusetts, Amherst. He has held visiting positions at the IBM T.J. Watson Research Center, Yorktown Heights, New York; Laboratoire MASI, Paris; INRIA, SophiaAntipolis, France; AT\&T Labs-Research, Florham Park, New Jersey; and Microsoft Research Lab, Cambridge, United Kingdom. His research interests include networks and performance evaluation. He currently serves as the editor-in-chief of the IEEE/ACM Transactions on Networking, and on the editorial boards of Journal of the ACM and the IEEE Journal on Selected Areas in Communications and has previously served on numerous other editorial boards. He was a program cochair of the Proceedings of the Joint International Conference on Measurement and Modeling of Computer Systems (SIGMETRICS '92/PERFORMANCE '92) conference and the PERFORMANCE '02 conference. He has received the 2007 IEEE Koji Kobayashi Award, the 2007 ACM SIGMETRICS Achievement Award, the 1998 IEEE Communications Society William Bennett Best Paper Award, and numerous conference/ workshop best paper awards. He is a member of ORSA and a fellow of the ACM and the IEEE.

Rene Cruz received the BSEE and PhD degrees from the University of Illinois at Urbana-Champaign in 1980 and 1987, respectively, and the SMEE degree from the Massachusetts Institute of Technology in 1982. $\mathrm{He}$ joined the faculty of the Department of Electrical and Computer Engineering, University of California, San Diego, La Jolla in 1987, where he is currently a professor of electrical and computer engineering. He is a fellow of the IEEE.

$\triangleright$ For more information on this or any other computing topic, please visit our Digital Library at www.computer.org/publications/dlib. 